\title{
A Model-free Approach to Automatic Dose Guidance in Long Acting Insulin Treatment of Type 2 Diabetes
}

Krishnamoorthy, Dinesh; Boiroux, Dimitri; Aradottir, Tinna Bjork; Engell, Sarah Ellinor; Jørgensen, John Bagterp

\section{Published in:}

IEEE Control Systems Letters

Link to article, DOI:

10.1109/LCSYS.2020.3047493

Publication date:

2021

Document Version

Peer reviewed version

Link back to DTU Orbit

Citation (APA):

Krishnamoorthy, D., Boiroux, D., Aradottir, T. B., Engell, S. E., \& Jørgensen, J. B. (2021). A Model-free Approach to Automatic Dose Guidance in Long Acting Insulin Treatment of Type 2 Diabetes. IEEE Control Systems Letters, 5(6), 2030-2035. https://doi.org/10.1109/LCSYS.2020.3047493

\section{General rights}

Copyright and moral rights for the publications made accessible in the public portal are retained by the authors and/or other copyright owners and it is a condition of accessing publications that users recognise and abide by the legal requirements associated with these rights.

- Users may download and print one copy of any publication from the public portal for the purpose of private study or research.

- You may not further distribute the material or use it for any profit-making activity or commercial gain

- You may freely distribute the URL identifying the publication in the public portal 


\title{
A Model-free Approach to Automatic Dose Guidance in Long Acting Insulin Treatment of Type 2 Diabetes*
}

\author{
Dinesh Krishnamoorthy ${ }^{1}$, Dimitri Boiroux ${ }^{2}$, Tinna Björk Aradóttir ${ }^{3}$, \\ Sarah Ellinor Engell ${ }^{2,3}$ and John Bagterp Jørgensen ${ }^{2}$
}

\begin{abstract}
This paper presents a model-free insulin titration algorithm for patients with type 2 diabetes that automatically finds and maintains the optimal insulin dosage in order to maintain the blood glucose concentration at desired levels. The proposed method is based on recursive least square based extremum seeking control. Since the proposed method does not require a detailed model, it can be applied on a wide population of patients without the need to identify and adapt models to the patient data. We demonstrate the effectiveness of the proposed method using in silico simulations, which are benchmarked against two standard-of-care approaches. We also show that the proposed method can handle intra-patient metabolic variations and non-adherence to the treatment regimen. Finally, using a population of $\mathbf{5 0}$ virtual patients, we show that the proposed method is able to handle inter-patient variations.
\end{abstract}

Index Terms-Healthcare and medical systems, Emerging control applications, Human-in-the-loop control

\section{INTRODUCTION}

D IABETES is a metabolic disorder characterized by a high blood glucose concentration. Type 1 diabetes (T1D) is due to absolute lack of insulin production by the pancreatic beta cells, which requires life-long insulin replacement therapy, e.g. using artificial pancreas (AP). Artificial pancreas uses continuous glucose measurement (sensor) and insulin infusion pumps (actuator) for glycemic regulation. Treatment of T1D using AP is a well studied problem by control engineers, leading to several advancements in AP technology [1]-[3], with a majority of literature on AP proposing different variants of MPC-based schemes, see for example [4]-[7] and the references therein.

However, relatively little attention has been provided to Type 2 diabetes (T2D) by the control community, despite

*This work was supported by the Research Council of Norway under the IKTPLUSS program (299585), and Innovation Fund Denmark in the project ADAPT-T2D (9068-00056B)

${ }^{1}$ Dinesh Krishnamoorthy is with the Department of Chemical Engineering, Norwegian University of Science and Technology, 7491, Trondheim, Norway dinesh.krishnamoorthy@ntnu.no

2 Dimitri Boiroux, Sarah Ellinor Engell and John Bagterp Jørgensen are with the Department of Applied Mathematics and Computer Science, Technical University of Denmark, DK-2800, kgs. Lyngby, Denmark dibo@dtu.dk, jbjo@dtu.dk

3 Tinna Björk Aradóttir and Sarah Ellinor Engell are with Novo Nordisk A/S, DK-2880, Bagsværd, Denmark tbao@novonordisk.com, sqee@novonordisk.com the fact that T2D accounts for around $90 \%$ of all the cases. T2D occurs due to an imbalance between insulin secretion and insulin resistance, leading to elevated blood glucose concentration over prolonged periods of time. T2D is a relatively more heterogeneous disorder than T1D, since different T2D patients may experience different levels of insulin resistance, and the insulin resistance also changes within the same patient with age, duration of diabetes, duration of insulin therapy, diet and physical activity etc. The heterogeneous nature of T2D makes it a relatively more challenging modelling and control problem.

The standard treatment procedure for T2D is commenced with changes to lifestyle such as diet and physical activity. This is often accompanied by non-insulin pharmaceuticals such as Metformin to increase insulin sensitivity. If this is still not sufficient to regulate the blood glucose concentration within the target range, one possibility is the insulin treatment. Typically, insulin treatment for T2D involves manually administering a dosage of long acting insulin (also known as basal insulin) e.g. using an insulin pen (actuator) once a day based on self-measured blood glucose (SMBG) readings, also recorded once a day (sensor) at fasting conditions. Finding a patient's optimal insulin dosage is known as insulin titration. Insulin titration is very important, since too low insulin may fail to lower the blood glucose concentration sufficiently, causing hyperglycemia, which leads to long term diabetic complications such as blindness, neuropathy etc. On the other hand, too high insulin dosage, leads to a severe short term effect, known as hypoglycemia, which can cause fainting, brain damage, or even death.

The standard-of-care clinical approach to an insulin titration procedure involves adjusting the insulin dosage by few units based on self-measured blood glucose (SMBG) data, measured daily at fasting conditions (i.e. pre-breakfast). However, in practice, the standard titration procedure is seldom followed, since the patients often do not feel comfortable/confident to adjust the dosage without the advice of a health care professional. In addition, the risk of hypoglycemia further undermines patient confidence.

From a control systems perspective, the insulin titration procedure can be seen as a feedback control problem, where the insulin dosage can be determined using an integral controller. In this paper, we consider the problem of insulin titration 
for patients with T2D. The main objective is to automatically adjust the long acting insulin dosage, based on the daily selfmeasured blood glucose (SMBG) data, to safely bring the fasting blood glucose concentration to a desired target.

Recently, automatic dose guidance in long acting insulin treatment for T2D using model predictive control (MPC) was proposed in [8]. Although the MPC-based solution provided superior performance than the standard clinical approach, it required relatively precise parameter values to be used in the prediction model. This work also considered an idealized case, where the MPC algorithm was initialized with the true physiological parameters. However, in practice, the MPC approach would require adapting the prediction model to each individual based on patient data, in addition to online state and parameter estimation. The use of an MPC-based artificial pancreas developed for T1D was also recently assessed for closed-loop insulin control in T2D [9], which also required updating the model parameters to each patient, and it was also concluded that the accuracy of the model predictions affected the actual target glucose concentration. The highly heterogeneous nature of $\mathrm{T} 2 \mathrm{D}$, thus makes it challenging to to use model-based control strategies.

To circumvent the modeling-related issues, we propose a model-free insulin titration approach, which is especially advantageous, given the heterogeneous nature of T2D. The proposed method is based on extremum seeking control. We show that the proposed model-free insulin titration approach is simple to use, and the model-free nature of the algorithm makes it applicable to a wide range of T2D patients without the need to tailor the algorithm or fit a model using patient data. Furthermore, we also show that the proposed algorithm can handle, inter- and intra-patient variations.

In the context of closed-loop insulin control, extremum seeking control has been previously studied only for target zone adjustment in the treatment of T1D, where the target range used by the MPC-based artificial pancreas is determined by an extremum seeking control layer above [10]. To the best of our knowledge, the use of extremum seeking control for insulin titration problems and for T2D has not been studied before in the literature. Furthermore, note that the authors in [10] considered the classical extremum seeking algorithm that is based on sinusoidal perturbation, which is not viable for T2D since the insulin is injected once day (i.e. actuation limitations). The fundamental differences in the sensing and actuation available in T1D and T2D limits what control algorithms can be used.

The main contribution of this paper is the application of recursive least square-based extremum seeking control to automatically titrate long acting insulin dosage for patients with T2D. The reminder of the paper is structured as follows. The proposed model-free insulin titration algorithm is presented in Section II. The virtual patient model used for the in silico simulations is presented in Section III. The standard-of-care approach used to benchmark the proposed method, and the results from the in silico simulation studies are presented in Section IV, before concluding the paper in Section V.

\section{MODEL-FREE INSULIN TITRATION ALGORITHM}

The main objective is to automatically adjust the long acting basal insulin dosage, $u(t)$, each day using only the daily self-measured blood glucose concentration $y(t)$ information (standard finger prick test), such that the fasting glucose level is steered to the target zone of $4-6 \mathrm{mmol} / \mathrm{L}$.

In order to automatically adjust the insulin dosage, we define a penalty function to steer the blood glucose concentration to a target of $5 \mathrm{mmol} / \mathrm{L}$, as shown below,

$$
J(y)=(y-5)^{2}+\rho(\min (0, y-5))^{2}
$$

The first term penalizes the deviations from the target of $5 \mathrm{mmol} / \mathrm{L}$. The second term mimics a soft constraint on the glucose level with a low limit of $5 \mathrm{mmol} / \mathrm{L}$, which is added to highly discourage low blood glucose concentration since this leads to hypoglycemia, which is undesirable.

The pre-breakfast SMBG measurement $y=f(u)$ can be assumed to be the steady-state blood glucose concentration as a function of the long acting insulin, and thus the penalty function $J(y)$ corresponds to the steady-state cost measurement. As mentioned earlier, the steady-state effect of long acting insulin on the pre-breakfast SMBG measurement $y=f(u)$ is unknown. Therefore, $J(f(u))$ can be seen as a static map between the cost function and the long acting insulin, which has a unique minimum $u^{*}$ due to the quadratic penalty function (1).

We now propose to use a recursive least square-based extremum seeking control to estimate the steady-state gradient $J_{u}$ of the static map, which is then driven to zero using an integral action. The SMBG measurement $y(k)$ obtained on the $k^{t h}$ day and the insulin dosage administered the previous day $u(k-1)$ are used to fit a local linear static function of the form,

$$
J(y(k))=\left[\begin{array}{ll}
u(k-1) & 1
\end{array}\right]\left[\begin{array}{l}
J_{u} \\
m
\end{array}\right]=\phi^{\top}(k) \theta(k)
$$

Note that the effect of insulin on the SMBG is not instantaneous and there is a measurement delay of 1 day, that is, the effect of the administered insulin is only observed in the SMBG measurements the following day. Therefore (2) fits $u(k-1)$ to $J(y(k))$.

When a new observation $y_{k}$ is available on the $k^{t h}$ day, the steady-state cost gradient $J_{u}(k)$ can be estimated online using a recursive least square algorithm as shown below [11],

$$
\begin{aligned}
\hat{\theta}(k) & =\hat{\theta}(k-1)+K(k)\left[J(y(k))-\phi^{\top}(k) \hat{\theta}(k-1)\right] \\
K(k) & =\frac{P(k-1) \phi(k)}{\lambda+\phi^{\top}(k) P(k-1) \phi(k)} \\
P(k) & =\frac{1}{\lambda}\left[P(k-1)-\frac{P(k-1) \phi(k) \phi^{\top}(k) P(k-1)}{\lambda+\phi^{\top}(k) P(k-1) \phi(k)}\right]
\end{aligned}
$$

where $\left[J(y(k))-\phi^{\top}(k) \hat{\theta}(k-1)\right]$ is the prediction error, $K(k)$ is the gain by how much each parameter will be modified in order to minimize the prediction error, and $P(k)$ is the covariance matrix. Since we are estimating a local linear model (2), the measurements obtained a long time ago does not reflect 


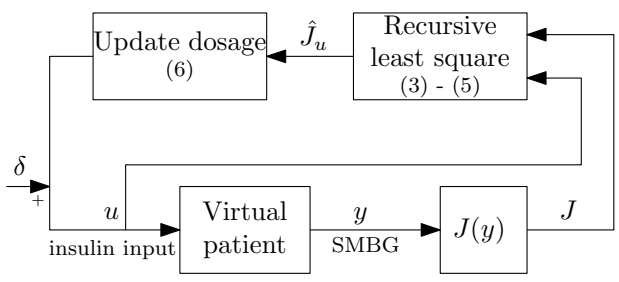

Fig. 1: Model-free Insulin titration algorithm based on recursive least squares estimation.

the current state of the system. Therefore, we introduce a forgetting factor $0<\lambda<1$, which allows the estimator to discount the effect of past measurements.

The insulin dosage is then updated using a gradient descent update $u(k)=u(k-1)+K_{I} \hat{J}_{u}(k)$ where $K_{I}$ is the adaptation gain. Fig. 1 schematically represents the proposed model-free insulin titration algorithm. For a large $K_{I}$, where the input can change rapidly, the forgetting factor $\lambda$ must be chosen relatively small, such that only the local effects are captured. As with any recursive algorithm, $\lambda$ is chosen such that the information dies away with a desired time constant of $1 /(1-\lambda)$ sample intervals, thereby using only the latest measurements where the local linear assumption holds. Rapid changes in insulin dosage from one day to another have been known to cause patient discomfort, and may even cause some diabetesrelated complications to worsen. Therefore, to avoid rapid dosage changes, we bound the rate of change of insulin, $\Delta u(k)=K_{I} \hat{J}_{u}$, between $\Delta u_{\min }$ and $\Delta u_{\max }$, yielding

$$
u(k)=u(k-1)+\max \left(\Delta u_{\min }, \min \left(\Delta u_{\max }, K_{I} \hat{J}_{u}\right)\right)
$$

Note that this also limits the effect of the tuning parameters such as integral gain. In order to ensure that the persistent excitation condition is satisfied, we add a small additional perturbation signal $\delta$ to the input, as shown in Fig. 1 .

\section{VIRTUAL PATIENT MODEL}

In this section, we present the virtual patient model used in the in silico study in this paper. Note that the model is only used to simulate the virtual patients. No knowledge of the model is used in the insulin titration algorithm.

We use a four compartment physiological model based on the Medtronic virtual patient (MVP) model [12] modified for T2D [8]. This model includes only the response of long acting insulin on fasting glucose, since this is what is captured in the SMBG measurements and this is also the desired timescale in which the controller is designed to perform. The insulin pharmacokinetics (PK) model describes the dynamics of insulin transition from the injection site to the plasma. The modified insulin PK model for T2D can be described as

$$
\begin{aligned}
\dot{I}_{s c}(t) & =\frac{1}{\tau}\left(\frac{u(t)}{C_{I}}-I_{s c}(t)\right) \\
\dot{I}_{p}(t) & =\frac{1}{\tau}\left(I_{s c}(t)-I_{p}(t)\right)
\end{aligned}
$$

where $u(t)$ is the exogenous long acting insulin input in [U/day], $I_{s c}$ and $I_{p}$ are the subcutaneous and plasma insulin concentrations in $[\mathrm{U} / \mathrm{L}], C_{I}$ is the insulin clearance rate in
[L/day], and $\tau$ is the insulin transition time constant [day]. In addition to the exogenously administered insulin $u$, the pancreas also produces insulin, which must be taken into account while modeling the insulin effect on blood glucose. Since the endogenous insulin production in the pancreas depends on the blood glucose concentration, this is modeled as a simple linear expression $I_{E N D O}(t)=\beta G(t)$, where $G(t)$ is the blood glucose concentration in [mmol/L] [8].

The pharmacodynamics (PD) model describes the combined effect of the exogenously administered insulin and the endogenous pancreatic insulin $\left(I_{p}(t)+I_{E N D O}(t)\right)$ on the blood glucose concentration, which is described by

$$
\begin{aligned}
\dot{I}_{e f f}(t) & =p_{2} S_{I}\left(I_{p}(t)+\beta G(t)\right)-p_{2} I_{e f f}(t) \\
\dot{G}(t) & =-\left(p_{G E Z I}+I_{e f f}(t)\right) G(t)+p_{E G P}+R_{a}
\end{aligned}
$$

where $I_{\text {eff }}(t)$ is the insulin effect on blood glucose [1/L], $S_{I}$ is the insulin sensitivity $[1 / \mathrm{U}], p_{E G P}$ is the rate of endogenous glucose production [mmol/L.day], $p_{2}$ is an inverse time constant that captures the delay in insulin action [1/day] and $p_{G E Z I}$ is an inverse time constant for the glucose to be eliminated from the plasma [1/day]. $R_{a}$ is the rate of appearance of glucose from meals [mmol/L.day]. However, since we only consider fasting glucose conditions, we do not include the effect of meal input, and set $R_{a}=0$.

The combined PK-PD model describing the effect of long acting insulin on the fasting blood glucose for patients with T2D can be rewritten by setting $\tilde{I}_{s c}=I_{s c} C_{I}, \tilde{I}_{p}=I_{p} C_{I}$, $\tilde{I}_{e f f}=I_{e f f} C_{I} / S_{I}$ and $\tilde{\beta}=\beta C_{I}$

$$
\begin{aligned}
\dot{\tilde{I}}_{s c}(t) & =\frac{1}{\tau}\left(u(t)-\tilde{I}_{s c}(t)\right) \\
\dot{\tilde{I}}_{p}(t) & =\frac{1}{\tau}\left(\tilde{I}_{s c}(t)-\tilde{I}_{p}(t)\right) \\
\dot{\tilde{I}}_{e f f}(t) & =p_{2}\left(\tilde{I}_{p}(t)+\tilde{\beta} G(t)\right)-p_{2} \tilde{I}_{e f f}(t) \\
\dot{G}(t) & =-\left(p_{G E Z I}+S_{I} \tilde{I}_{e f f}(t)\right) G(t)+p_{E G P}
\end{aligned}
$$

The ODE is re-written as a stochastic differential equation (SDE) by adding the diffusion term $\sigma d \omega(t)$ in order to capture the day-to-day glucose variations.

$$
\mathrm{d} \mathbf{x}(t)=f(\mathbf{x}(t), u(t), \mathbf{p}(t)) \mathrm{d} t+\sigma d \omega(t)
$$

where $\mathbf{p}:=\left[\tau, p_{2}, p_{G E Z I}, p_{E G P}, \tilde{\beta}, S_{I}\right]^{\top}$ are the model parameters, and $\mathbf{x}:=\left[\tilde{I}_{s c}, \tilde{I}_{p}, \tilde{I}_{e f f}, G\right]^{\top}$ are the states.

Long acting insulin dosage $u(t)$ is administered to the virtual patient and the SMBG data measures the fasting blood glucose concentration $y(t)=G(t)$. To simulate the virtual patient, the SDE (15) was solved numerically using the EulerMaruyama method, where the sampling interval is divided into $N$ equal sub-interval of width $\Delta t>0$. Starting with the initial condition of $\mathbf{x}(0)$, the SDE is numerically solved as

$$
\begin{gathered}
\mathbf{x}\left(\tau_{k}\right)=\mathbf{x}\left(\tau_{k-1}\right)+f\left(\mathbf{x}\left(\tau_{k-1}\right), u\left(\tau_{k-1}\right), \mathbf{p}\left(\tau_{k-1}\right)\right) \Delta t \\
+\sigma \Delta W_{k}
\end{gathered}
$$

with $\Delta W_{k} \sim \mathcal{N}(0, \Delta T)$. In this paper, we choose a sampling time of $1 \mathrm{~h}$ divided equally into $N=10$ sub-intervals of equal width $\Delta t=6 \mathrm{~min}$. The variance $\sigma$ was varied in the 
simulations to simulate patients with different levels of day-today glucose variations [8]. The main motivation behind using the modified MVP model for the in silico study is that we are mainly interested in the slow dynamics of basal insulin on fasting glucose (captured by the sparse SMBG data). This model has been developed and validated using sparse clinical data, see [13], [14] and the pertinent references therein.

\section{SIMULATION RESULTS}

We test the proposed model-free insulin titration algorithm using in silico simulations using the virtual patient described by (15). The time constant was fixed at $\tau=12 \mathrm{~h}$ based on the time to maximum glucose infusion rate published in [15]. Based on the mean values published in [12], the inverse time constants were set to $p_{2}=15.8 \mathrm{day}^{-1}$ and $p_{\text {GEZI }}=3.31 \mathrm{day}^{-1}$. The remaining parameters were varied to simulate different virtual patients.

To benchmark the proposed algorithm, we implement the standard-of-care approach to insulin titration, which is a simple paper-based algorithm that adjust the dose by a few units using a look-up table. In this paper, we compare with the simple "202" titration algorithm as well as the stepwise algorithm used in the clinical trial of insulin degludec for patients with T2D [16]. In this approach, the dose is updated once a week, and the dose change is based on a the SMBG measurement. In the simple 202 method, the insulin dosage is either increased or decreased by $2 \mathrm{U}$ depending on a single SMBG measurement according to the look-up table shown in Table I. Whereas, in the stepwise method, the insulin dose in updated once a week based on the average SMBG measured over the past three days, according to the look-up table shown in Table I. If any hypoglycemic event is recorded, then the dosage is reduced by $2 \mathrm{U}$. Note that both the standard-of-care methods rely on absolute SMBG measurements, as opposed to our proposed algorithm which is based on the gradient, that is change in SMBG per unit change in the insulin.

TABLE I: Standard-of-care titration algorithm from [16].

\begin{tabular}{l|c|c}
\hline Method & SMBG $[\mathrm{mmol} / \mathrm{L}]$ & Dose adjustment $\Delta u[\mathrm{U}]$ \\
\hline \multirow{3}{*}{202} & $>6$ & +2 \\
& $4-6$ & No change \\
& $<3.9$ & -2 \\
\hline \multirow{5}{*}{ stepwise } & $>9$ & +8 \\
& $8-8.9$ & +6 \\
& $7-7.9$ & +4 \\
& $5-6.9$ & +2 \\
& $3.9-4.9$ & No change \\
& $3.1-3.8$ & -2 \\
\hline
\end{tabular}

We first simulate a single virtual patient with $S_{I}=1.8$ $\mathrm{U}^{-1}, p_{E G P}=368 \mathrm{mmol} / \mathrm{L} /$ day and $\tilde{\beta}=1.68 \mathrm{UL} / \mathrm{mmol} /$ day. For the stochastic simulations, we set the variance to $\sigma=2 I$ in order to capture the average day-to-day glucose variations as reported in literature [8]. The initial states of the virtual patient are set to $\mathbf{x}(0)=\left[\tilde{I}_{s c}, \tilde{I}_{p}, \tilde{I}_{e f f}, G\right]^{\top}=[0,0,15.2,12]^{\top}$, with an initial insulin dosage of $u(0)=10 \mathrm{U}$. We simulate the virtual patient for a total of 60 days.

The weighting factor for hypoglycemic blood glucose concentrations in the penalty function (1) is set to $\rho=8$. A
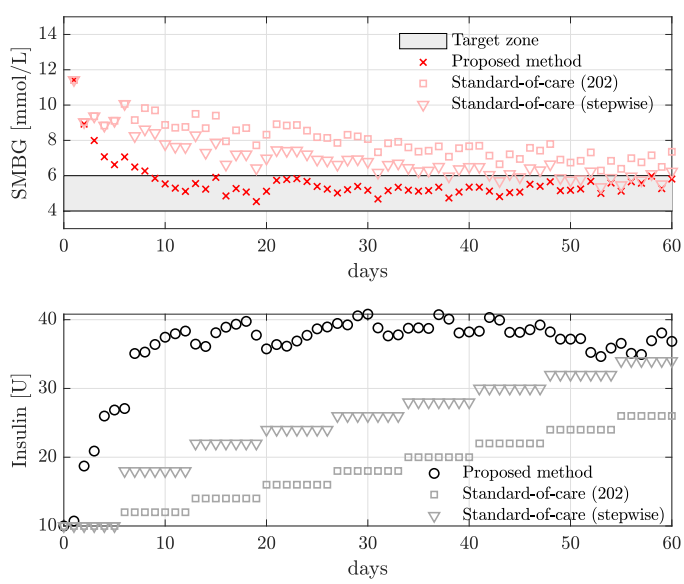

Fig. 2: Simulation results showing the performance of the proposed RLS-based model-free insulin titration algorithm compared with the standard-of-care approach.

TABLE II: Performance of the proposed approach compared to the standard-of care approaches.

\begin{tabular}{c|ccc}
\hline & $\begin{array}{c}\text { Proposed } \\
\text { method }\end{array}$ & $\begin{array}{c}\text { SOC } \\
\text { (stepwise) }\end{array}$ & $\begin{array}{c}\text { SOC } \\
(202)\end{array}$ \\
\hline Cumulative cost & 319.566 & 542.676 & 838.59 \\
Average SMBG [mmol/L] & 5.541 & 6.853 & 7.896 \\
no. of days to target & $\sim 10$ & $\sim 40$ & $>60$ \\
\hline
\end{tabular}

forgetting factor of $\lambda=0.01$ is used in the recursive least squares estimation. The integral gain in (6) is set to $K_{I}=$ 1500 , and the maximum and minimum dosage change each day is limited to $\Delta u_{\min }=\Delta u_{\max }=8 \mathrm{U}$ when outside the target, and $\Delta u_{\min }=\Delta u_{\max }=2 \mathrm{U}$ when the glucose reading is within the target. These values are aligned with the stepwise standard-of-care method. In order to ensure persistence of excitation, we add a small additional dose of $\delta= \pm 0.5 \mathrm{U}$ to the computed insulin dosage. The simulations were performed using MATLAB v2019b.

\section{A. Insulin Titration}

First we test the performance of the proposed model-free method and compare it with the standard-of-care approaches. In both the cases, the insulin titration is initiated at $10 \mathrm{U}$ insulin. Fig. 2 shows the simulation results, which illustrates the that proposed model-free insulin titration algorithm (shown in red cross) is able to successfully bring the blood glucose concentration to the target zone of $4 \mathrm{mmol} / \mathrm{L}-6 \mathrm{mmol} / \mathrm{L}$, within approximately 10 days from the start of insulin titration. Whereas with the stepwise standard-of-care approach (shown in light red triangle) takes more than a month to reach the target zone, and with the 202 standard-of-care (shown in light red square), the blood glucose concentration is not within the target zone even after 60 days. The corresponding insulin doses are shown in the bottom subplot. The cumulative cost of glycemic variation over 60 days obtained by integrating (1), and the average glucose readings of the three approaches are summarized in Table II. 

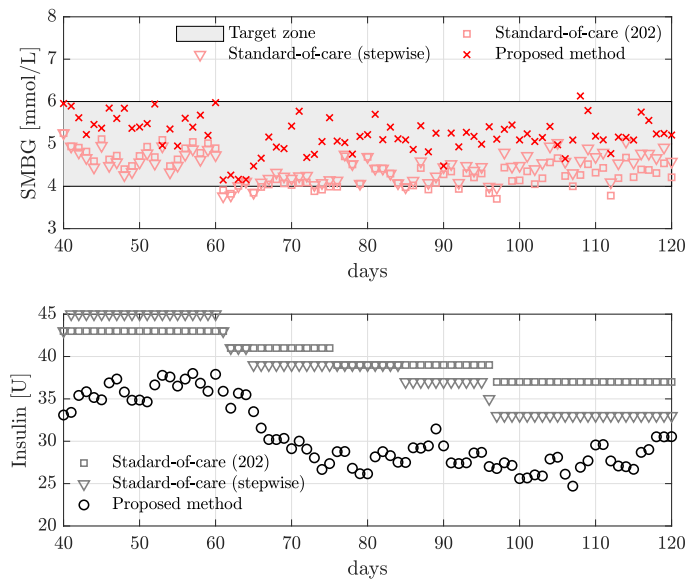

Fig. 3: Intra-patient metabolic variations: Simulation results showing the insulin dosage adjustment when insulin sensitivity $S_{I}$ increases by $30 \%$ on day 60 .

\section{B. Variations in Insulin Sensitivity}

We now test the performance of the algorithm to handle changes in the physiological state of the patient (intra-patient variations), which may be due to stress, physical activity, diet, weight loss etc. We simulate a case for the same virtual patient as above, where the insulin sensitivity $S_{I}$ increases on day 60 by $30 \%$. The increased insulin sensitivity would require a reduced insulin dosage in order to avoid hypoglycemic events. The simulation starts with the blood glucose concentration close to the target zone, with a corresponding initial insulin dosage of $35 \mathrm{U}$.

Fig. 3 clearly shows that as the insulin sensitivity increases, the insulin dosage is reduced accordingly in order to avoid hypoglycemic events (glucose concentration $<4 \mathrm{mmol} / \mathrm{L}$ ). Fig. 3 also shows the standard-of-care approaches, where it can be seen that 6 hypoglycemic events occurred with the stepwise algorithm, with a couple of hypoglycemic events occurring 1 month after the change in the insulin sensitivity. With the 202 approach, more than 10 hypoglycemic events occurred between days 60 and 120. This illustrates the that proposed model-free insulin titration algorithm is able to successfully adjust the dosage despite variations in the insulin sensitivity.

\section{Non-adherence}

The insulin titration relies on the self-measured blood glucose data, which is sampled manually at pre-breakfast conditions. Therefore, adherence to the treatment regimen is expected from the patients, making this a cyber-physicalhuman system (CPHS). However, it may happen that the patient, for whatever reason, forgets to measure the prebreakfast blood glucose concentration on some days, and usually also forgets to inject the insulin dosage on the same day. It is therefore important that the insulin titration algorithm is robust to such non-adherence.

In order to test the proposed algorithm in the case of nonadherence to the treatment regimen, we simulate a case where the patient forgets to record the SMBG measurement and
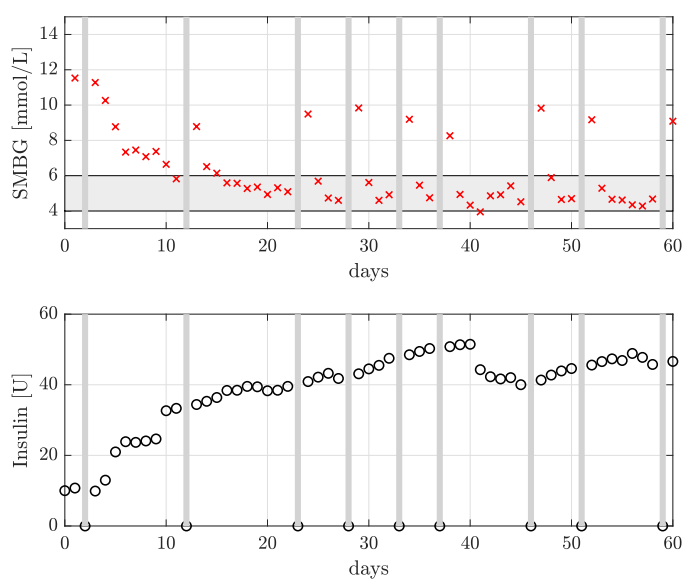

Fig. 4: Effect of non-adherence: Simulation results with nonadherence, where gray solid lines indicate the days with missing SMBG data and forgotten insulin injection.

inject insulin on some days chosen randomly. We use the same virtual patient as in the previous cases. Fig. 4 shows the simulation results, where the gray bar indicates the days with non-adherence. It can be clearly seen that the proposed algorithm is able to bring the blood glucose concentration to the target zone despite repeated non-adherence to treatment regimen.

\section{Inter-patient variations}

One of the main advantages of using a model-free insulin titration method is that it does not require adapting the models to individual patients, unlike previously studied methods [8], [9]. In this section, we test the wide applicability of the proposed algorithm on a population of 50 virtual patients with T2D, each with different levels of insulin sensitivity, endogenous glucose production rates, and insulin production rates. We choose a population of 50 patients, where the insulin sensitivity for the $i^{t h}$ patient is randomly chosen from $S_{I}^{(i)} \in[1,2.5] \mathrm{U}^{-1}$, endogenous glucose production is randomly chosen from $p_{E G P}^{(i)} \in[350,380] \mathrm{mmol} / \mathrm{L} /$ day , and the insulin production co-efficient is randomly chosen from $\tilde{\beta}^{(i)} \in[1.1,1.5] \mathrm{UL} / \mathrm{mmol} / \mathrm{day}$. The different patients may also have a different levels of day-to-day glycemic variations. In order to reflect this, the variance used in the stochastic simulations, $\sigma$ is also randomly chosen for each patient from a distribution of $\sigma \sim \mathcal{N}(2,1)$.

For all the 50 virtual patients, we use the same algorithm with identical controller tuning parameters. In all the patients, the titration begins from an initial dosage of 10 U. Fig. 5 shows the daily pre-breakfast blood glucose concentration for each patient, where it can be clearly seen that the SMBG data for all the 50 patients are successfully brought to the target zone within 2 weeks from the start of insulin titration. The histogram (bottom left subplot) also shows that $75.45 \%$ of the SMBG measurements recorded in all the 50 patients were within the target range of $4 \mathrm{mmol} / \mathrm{L}-6 \mathrm{mmol} / \mathrm{L}$ after 10 days from the start of insulin titration. The bottom right subplot 

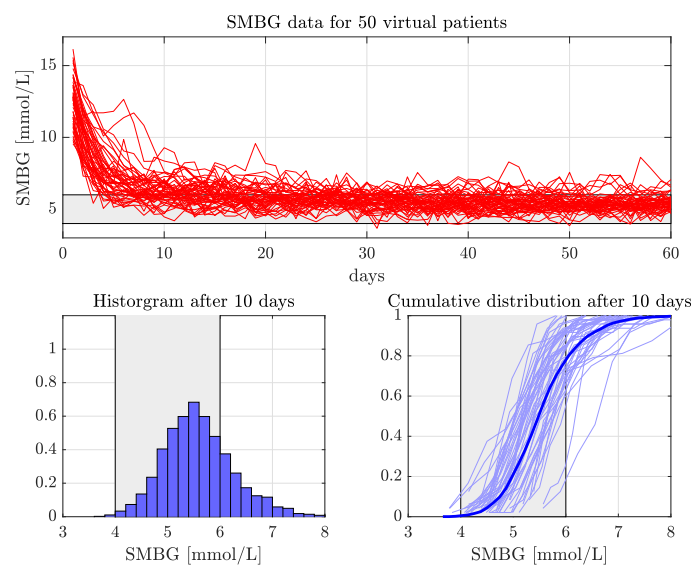

Fig. 5: Inter-patient variations: (top subplot) Evolution of SMBG for a population of 50 patients using the proposed model-free insulin titration approach. (Bottom subplots) Histogram and the cumulative distribution of the SMBG measurements of all the 50 patients and the average, 10 days after the start of insulin titration.

TABLE III: Percentage of time spent by the population of 50 patients in different blood glucose concentration ranges.

\begin{tabular}{c|ccc}
\hline SMBG & $\begin{array}{c}\text { Severe } \\
\text { hypoglycemia }\end{array}$ & Hypoglycemia & Time-in-target \\
in [mmol/L] & $<3$ & $3-4$ & $4-6$ \\
\hline All data points & $0 \%$ & $0.197 \%$ & $63.93 \%$ \\
10 days after & $0 \%$ & $0.25 \%$ & $75.45 \%$ \\
\hline
\end{tabular}

shows the cumulative distribution of the individual patients, as well as the average cumulative distribution. Table III shows the percentage of time spent by the 50 patients in different blood glucose concentration ranges, from the start of insulin titration, as well as 10 days after insulin titration.

More than the convergence time, safety of the dose guidance is of primary importance, where we want to avoid hypoglycemic events. From Table III we can see that no severe hypoglycemic events $(S M B G<3 \mathrm{mmol} / \mathrm{L})$ occurred using our proposed algorithm. We do not report hyperglycemic (SMBG between $10-13.9 \mathrm{mmol} / \mathrm{L}$ ) and sever hyperglycemic (SMBG $>13.9 \mathrm{mmol} / \mathrm{L}$ ) events here, since these typically stem from post-prandial excursions, which are not considered in this study. Even otherwise it can be clearly seen from Fig. 5 that no hyperglycemic events were observed in the SMBG measurements, indicating good long-term fasting glucose control.

\section{CONCLUSIONS}

This paper addresses the problem of determining and maintaining the optimal insulin dosage for T2D patients in a model-free fashion. The proposed method uses recursive least squares-based extremum seeking control. We showed that the proposed model-free algorithm is able to determine the optimal insulin dosage using only the daily self-measured blood glucose (SMBG) data. More importantly, this method does not require updating models or fit model parameters to individual patients, thus making it widely applicable to a large group of patients. Compared to the standard-of-care approach, the proposed method was shown to perform better in terms of days-to-convergence and reduce hypoglycemic events. This paper is a step towards model-free algorithms for insulin titration in T2D, and data-driven closed-loop glycemic regulation. Future work will involve testing the proposed algorithm on a more detailed physiological model that have recently been developed for T2D such as [17], [18]. The proposed method may also be used for model-free basal insulin titration for patients in T1D.

\section{REFERENCES}

[1] H. Thabit and R. Hovorka, "Coming of age: the artificial pancreas for type 1 diabetes," Diabetologia, vol. 59, no. 9, pp. 1795-1805, 2016.

[2] C. Cobelli, C. Dalla Man, G. Sparacino, L. Magni, G. De Nicolao, and B. P. Kovatchev, "Diabetes: models, signals, and control," IEEE reviews in biomedical engineering, vol. 2, pp. 54-96, 2009.

[3] K. Turksoy and A. Cinar, "Adaptive control of artificial pancreas systems-a review," Journal of healthcare engineering, vol. 5, no. 1, pp. $1-22,2014$.

[4] L. Magni, D. M. Raimondo, L. Bossi, C. D. Man, G. D. Nicolao, B. Kovatchev, and C. Cobelli, "Model predictive control of type 1 diabetes: An in silico trial," Journal of Diabetes Science and Technology, vol. 1, no. 6, pp. 804-812, 2007.

[5] R. Gondhalekar, E. Dassau, H. C. Zisser, and I. Francis J. Doyle, "Periodic-zone model predictive control for diurnal closed-loop operation of an artificial pancreas," Journal of Diabetes Science and Technology, vol. 7, no. 6, pp. 1446-1460, 2013.

[6] R. Hovorka, V. Canonico, L. J. Chassin, U. Haueter, M. Massi-Benedetti, M. O. Federici, T. R. Pieber, H. C. Schaller, L. Schaupp, T. Vering et al., "Nonlinear model predictive control of glucose concentration in subjects with type 1 diabetes," Physiological measurement, vol. 25, no. 4, p. 905, 2004.

[7] D. Boiroux, D. A. Finan, J. B. Jørgensen, N. K. Poulsen, and H. Madsen, "Nonlinear model predictive control for an artificial $\beta$-cell," in Recent Advances in Optimization and its Applications in Engineering. Springer, 2010, pp. 299-308.

[8] T. B. Aradóttir, D. Boiroux, H. Bengtsson, J. Kildegaard, M. L. Jensen, J. B. Jørgensen, and N. K. Poulsen, "Model predictive control for dose guidance in long acting insulin treatment of type 2 diabetes," IFAC Journal of Systems and Control, vol. 9, p. 100067, 2019.

[9] H. Thabit, S. Hartnell, J. M. Allen, A. Lake, M. E. Wilinska, Y. Ruan, M. L. Evans, A. P. Coll, and R. Hovorka, "Closed-loop insulin delivery in inpatients with type 2 diabetes: a randomised, parallel-group trial," The Lancet Diabetes \& Endocrinology, vol. 5, no. 2, pp. 117-124, 2017.

[10] Z. Cao, R. Gondhalekar, E. Dassau, and F. J. Doyle, "Extremum seeking control for personalized zone adaptation in model predictive control for type 1 diabetes," IEEE Transactions on Biomedical Engineering, vol. 65, no. 8, pp. 1859-1870, 2017.

[11] L. Ljung, System identification: theory for the user, 2nd ed. Upper Saddle River, NJ: PTR Prentice Hall, 1999.

[12] S. S. Kanderian, S. Weinzimer, G. Voskanyan, and G. M. Steil, "Identification of intraday metabolic profiles during closed-loop glucose control in individuals with type 1 diabetes," 2009.

[13] T. B. Aradóttir, D. Boiroux, H. Bengtsson, and N. K. Poulsen, "Modelling of fasting glucose-insulin dynamics from sparse data," in 2018 40th Annual International Conference of the IEEE Engineering in Medicine and Biology Society (EMBC). IEEE, 2018, pp. 2354-2357.

[14] _ "Modelling of glucose-insulin dynamics from low sampled data," IFAC-PapersOnLine, vol. 51, no. 15, pp. 551-556, 2018.

[15] T. Heise, L. Nosek, S. Bøttcher, H. Hastrup, and H. Haahr, "Ultra-longacting insulin degludec has a flat and stable glucose-lowering effect in type 2 diabetes," Diabetes, Obesity and Metabolism, vol. 14, no. 10, pp. 944-950, 2012.

[16] T. Kadowaki, H. Jinnouchi, K. Kaku, M. L. Hersløv, J. Hyllested-Winge, and S. Nakamura, "Insulin degludec in a simple or stepwise titration algorithm in a japanese population of patients with type 2 diabetes: a randomized, 26-week, treat-to-target trial," Diabetology international, vol. 8, no. 1, pp. 87-94, 2017.

[17] R. Visentin, C. Cobelli, and C. Dalla Man, "The padova type 2 diabetes simulator from triple-tracer single-meal studies: In silico trials also possible in rare but not-so-rare individuals," Diabetes Technology \& Therapeutics, 2020.

[18] M. A. Ahdab, J. Leth, T. Knudsen, and H. Clausen, "Glucoseinsulin dynamical model for type 2 diabetic patients," arXiv preprint arXiv:2008.01614, 2020. 